\title{
Presence of Chlamydia Infection among asymptomatic Female Commercial Sex-workers (CSWs) in the Kumasi Metropolis, Ghana
}

\author{
Amina Abubakari ${ }^{1,2}$, Samuel N. Osei-Djarbeng ${ }^{3 *}$, John Asiedu Larbi ${ }^{1}$ and \\ Enoch H. Frimpong
}

${ }^{1}$ Department of Theoretical and Applied Biology, College of Sciences, Kwame Nkrumah University of Science and Technology, KNUST, Kumasi, Ghana

${ }^{2}$ Department of Laboratory Technology, Faculty of Medicine and Health Sciences, Kumasi

Polytechnic, Kumasi, Ghana

${ }^{3}$ Department of Pharmaceutical Sciences, Faculty of Medicine and Health Sciences, Kumasi Polytechnic, Kumasi, Ghana

${ }^{4}$ Department of Clinical Microbiology, College of Health sciences, Kwame Nkrumah University of Science and Technology, KNUST, Kumasi, Ghana

*Corresponding author

Keywords

Commercial, Sex workers, Kumasi, Asymptomatic, Chlamydia

\section{Article Info}

Accepted:

16 December 2015

Available Online:

10 January 2016
Distribution and prevalence of Chlamydia infection among asymptomatic female commercial sex-workers (CSWs) in Kumasi was studied using the Rapid Immunoassay Chlamydia Test. The study targeted women (CSWs) aged 18 to 35 years who were actively engaged in sex work. Endocervical swabs were collected from each of the hundred (100) participants who consented to take part in the study. The QuickVue Chlamydia test was used after a pre-tested questionnaire had been administered to capture demographic data. The prevalence of Chlamydia infection was $19.0 \%$. The highest prevalence was recorded among the age group 18-22 years, while low prevalence was found among those above 30 years. The high presence of Chlamydia trachomatis among the asymptomatic CSWs highlights their role in transmission and thus calls for STI education as well as routine screening among sex workers.

\section{Introduction}

Chlamydia infection is a sexually transmitted infection (STI) caused by an obligate intracellular parasite called Chlamydia trachomatis (Mishori et al., 2012). An estimated 3 million infections occur annually among sexually active adolescents and young adults in the United
States (Coombes, 2004). The prevalence in Africa is low compared to the western world, which could be attributed to the more rampant use of unprescribed antibiotics such as doxycycline, azithromycin, erythromycin, tetracycline and oxytetracycline (Coombes, 2004). In 1988, a study conducted in 
Kumasi (Ghana) reported a prevalence of $3.6 \%$ for Chlamydia infection for patients attending prenatal care and infertility clinic, using the RNA Chlamydia Kit (Drescher et al., 1988). Similarly, in 2004, another study was conducted in Korle-Bu Teaching Hospital, Accra, Ghana, on the prevalence of STIs and that of Chlamydia trachomatis was recorded as $3.0 \%$ when immunofluorescent monoclonal antibody technique was used (Apea-Kubi et al., 2004). Similar studies have been carried out in Benin, Gambia and Cameroon, all in Africa (Buve et al., 2001; Ngandjio et al., 2003). These studies showed different prevalence rates.

Research into the prevalence of Chlamydia infection is important, as STIs are some of the major problems the world is facing. The majority of persons with Chlamydia infec tion are not aware of the infection because they do not have symptoms (asymptomatic). Because of this sexually active people transmit the infection unknowingly. Screening, therefore, is necessary to identify and treat those infected (Mishori, 2001).

Genital Chlamydia infection in women has serious sequelae. This is because untreated Chlamydia infection can persist for a long period of time, and up to $30 \%$ of inadequately treated women may go on to develop pelvic inflammatory diseases PID (Bolam et al., 1997).

It has been estimated that up to half of all cases of PIDs in developing countries can be attributed to Chlamydia. Among those with symptomatic PID, one fifth may become infertile and one tenth may suffer ectopic pregnancy (Simms et al., 1998).

Epidemiological studies suggest that Chlamydia infection also confer increased risk for cervical squamous cell carcinoma
(SCC) (Anttila et al., 2001). Due to the asymptomatic nature of Chlamydia infection routine screening is not usually done hence periodic studies must be carried out to determine the prevalence. This current study involved asymptomatic female commercial sex workers and symptomatic female nonsex workers presenting for STI at Komfo Anokye Teaching Hospital (KATH) in Kumasi, Ghana.

Symptoms of both Chlamydia and Gonococcus infections are similar, therefore the latter is normally investigated when a person shows symptoms of Chlamydia infection which include discharge, frequent urge to urinate. In this study gonococci infection was also investigated among the study subjects.

The objectives of the study therefore are to determine the prevalence of Chlamydia infection among asymptomatic female commercial sex-workers (CSWs) and also to find out the prevalence of symptomatic female non-commercial sex workers (nonCSWs) presenting with STIs at KATH. Additionally, the prevalence of Gonococcus infection among the mentioned groups was also determined.

\section{Materials and Methods}

\section{Study Area}

The study was conducted at the Microbiology Laboratory of Komfo Anokye Teaching Hospital (KATH), Kumasi. The Hospital is well patronized due to its location in the city center of Kumasi, and also being the second largest Hospital in Ghana after Korle-Bu Teaching Hospital in Accra, Ghana. It is open to patients from all walks of life for 24 hours a day. 


\section{Study Subjects}

One hundred and fifty females comprising 100 commercial sex workers (CSWs) who are asymptomatic for Chlamydia infection, and 50 symptomatic non-commercial sex workers (non-CSWs) used as a control group were used in the study. The study considered CSWs with NO symptoms of Chlamydia infection; those who showed symptoms of STIs particularly that of Chlamydia were not included in the study. In the case of the non-CSWs, those who showed symptoms were the only ones employed in the study.

\section{Ethical Clearance and Study Subjects Consent}

Ethical clearance was sought from the ethical clearance committee. In addition, verbal consent was also sought from both the CSWs and non-CSWs. They were educated about the purpose of the study, and they had the right discontinue from taking part in the study at any time. The participants were, further, made aware that all data obtained from them would be confidential and once the required information had been collated and the results published it would be destroyed.

\section{Sample Collection and Processing}

A well-designed questionnaires containing biographic data of participants were administered to each of the women recruited for the study. After completion of the questionnaires, and with their permissions vaginal swabs were taken for analysis.

Two swabs were taken after initial removal of excess mucus from the exo-cervix. These were taken from the endo-cervix and were analyzed for presence or otherwise of Chlamydia and Gonococcus. The specimens were tested immediately after collection.

\section{Sample Preparation}

QuickVue Chlamydia test (Plate 1), which contains reagent A (Extraction solution), reagent $\mathrm{B}$ (Neutralization solution) and a cassette, was employed for investigation of the Chlamydial infection.

Reagent A was used to extract the endocervical specimen taken from the subjects; followed by Reagent B as a neutralizing solution. Three (3) drops of the prepared sample were added to the well in the cassette. The sample migrated through a label pad containing a monoclonal antiChlamydia antibody conjugated with a pinkColorado test label and a blue-coloured label. If the sample contained Chlamydia antigen, the antigen bound to the antibody coupled to the pink coloured test label which in turn, bound to a second monoclonal antiChlamydia LPS antibody on the membrane. If Chlamydia antigen-antibody complex was captured, a faint to dark red test line would be visible. A blue control line also appeared in the result window indicating that right volume of clinical sample entered the test cassette and capillary flow occurred. If Chlamydia antigen was not present only a blue control line would be visible.

One of the swabs was used for the Chlamydia test and the other, for Gonococcal test.

For the Gonococcal test, Gram staining was first done, and when found to be Gramnegative, it was then cultured on Thayer Martin's medium for Gonococcus for confirmation (Thayer and Martin, 1966).

\section{Statistical Analysis}

Chi-square table and confidence intervals between the two proportions (asymptomatic and symptomatic) were used to analyze the result statistically. 


\section{Results and Discussion}

Of the 100 asymptomatic female commercial sex workers screened $56 \%$ were between the ages 18-22, 29\% between the ages $23-27,8 \%$ were also between the ages 28-32 and finally those who were between the ages of $33-37$ were $7 \%$. None of the asymptomatic female commercial sex workers were married nor widowed, rather they were all single. On their educational background, $59 \%$ were literate and $41 \%$ were completely illiterate. Nineteen (19) \% of them were students in different institutions, $36 \%$ were traders, none of them was a housewife, $5 \%$ were professionals in different professions and $40 \%$ of the women were engaged in purely sex-work.

In the case of the 50 symptomatic female non-sex workers screened, the age ranges from 18-22 and 23-27 years each recorded $28 \%$; $24 \%$ were between the ages $28-32$; and $20 \%$ between the ages of 33-37 years. Fifty six $(56 \%)$ of the symptomatic female non-sex workers screened were married, $40 \%$ were single and $4 \%$ were widowed. On their educational background, $56 \%$ were educated and $44 \%$, illiterate. Ten $(10 \%)$ of the symptomatic females screened were professionals with various professions; $22 \%$ were students in various institutions, $60 \%$ were traders and $8 \%$ were housewives. The above results are represented in Tables 1 and 2, and Figure 1.

Among the 100 asymptomatic female commercial sex-workers screened, $19.0 \%$ were positive to Chlamydia infection and only $1.0 \%$ was positive for Gonococcus infection. With the 50 symptomatic female non-sex workers screened $8.0 \%$ showed positive results to Chlamydia infection and none of them was positive for Gonococcus as presented in Table 3.
The current study has revealed a high prevalence of Chlamydia infection among asymptomatic CSWs. In a previous study which was carried out by Apea-Kubi and colleagues in 2004 at Korle-Bu Teaching Hospital, Accra-Ghana, a prevalence of $3.0 \%$ was recorded for Chlamydia infection among 517 women screened. A similar study conducted by Ngandjio and others in 2003 documented a prevalence of $3.96 \%$ for Chlamydia infection in Cameroon among 1277 volunteer students. The current study focused on commercial sex-workers presenting no symptoms of Chlamydia; the studies referred to (Apea-Kubi et al, 2004 and Ngandjio et al., 2003) focused on women in general. This may explain why they recorded lower prevalence than the current study did. CSWs sleep with multiple partners sometimes without protection against possible sexually transmitted infections (STIs), and this increases their chances of acquiring Chlamydia infection. Chlamydia infection, because it may be asymptomatic in both males and females, are mostly left untreated and this situation has been implicated as a major cause of tubal infertility and ectopic pregnancy in women (CDCP, 1993).

Interestingly, the study showed that noncommercial sex workers (non-CSWs) who rather presented 'symptoms' of Chlamydia to the hospital, when screened showed that only $8 \%$ were actually positive for Chlamydia. The non-CSWs, perhaps, mistakenly took the symptoms of other disease conditions such as candidiasis and even normal vaginal discharge to be symptoms of Chlamydial infection. The results obtained for the non-CSWs has shown that more education needs to be carried out; women need to know more precisely what the actual symptoms and signs of Chlamydia are. In Ghana where one can easily acquire antibiotics without 
prescription, if proper diagnosis is not carried out, people would wrongly administer antibiotics apparently to treat a non-existing infection.
Indiscriminate use of antibiotics worsen the already existing problem of antibiotic resistance (Sahoo et al., 2010).

Table.1 Age Range of the Subjects (CSWs and non-CSWs) Recruited for the Study

\begin{tabular}{llr}
\cline { 2 - 3 } Age (years) & CSWs & non-CSWs \\
\hline $18-22$ & $56(56 \%)$ & $14(28 \%)$ \\
$23-27$ & $29(29 \%)$ & $14(28 \%)$ \\
$28-32$ & $8(8 \%)$ & $12(24 \%)$ \\
$33-37$ & $7(7 \%)$ & $10(20 \%)$ \\
\hline
\end{tabular}

Table.2 Marital Status of the Subjects (CSWs and non-CSWs) Recruited for the Study

\begin{tabular}{lcc}
\cline { 2 - 2 } Marital status & CSWs & non-CSWs \\
\hline Married & $0(0 \%)$ & $28(56 \%)$ \\
Single & $100(100 \%)$ & $20(40 \%)$ \\
Widow & $0(0 \%)$ & $2(4 \%)$ \\
& &
\end{tabular}

Table.3 Prevalence of Chlamydial and Gonococcal Infections among the Subjects

\begin{tabular}{llcc}
\hline & & \multicolumn{2}{c}{ Positives } \\
\cline { 3 - 4 } Subject Group & Total Number & Chlamydia & Gonococcus \\
\hline CSWs & 100 & $19(19 \%)$ & $1(1 \%)$ \\
Non-CSWs & 50 & $4(8 \%)$ & $0(0 \%)$ \\
\hline
\end{tabular}

Figure.1 Frequencies of other Activities Engaged by the Women Screened

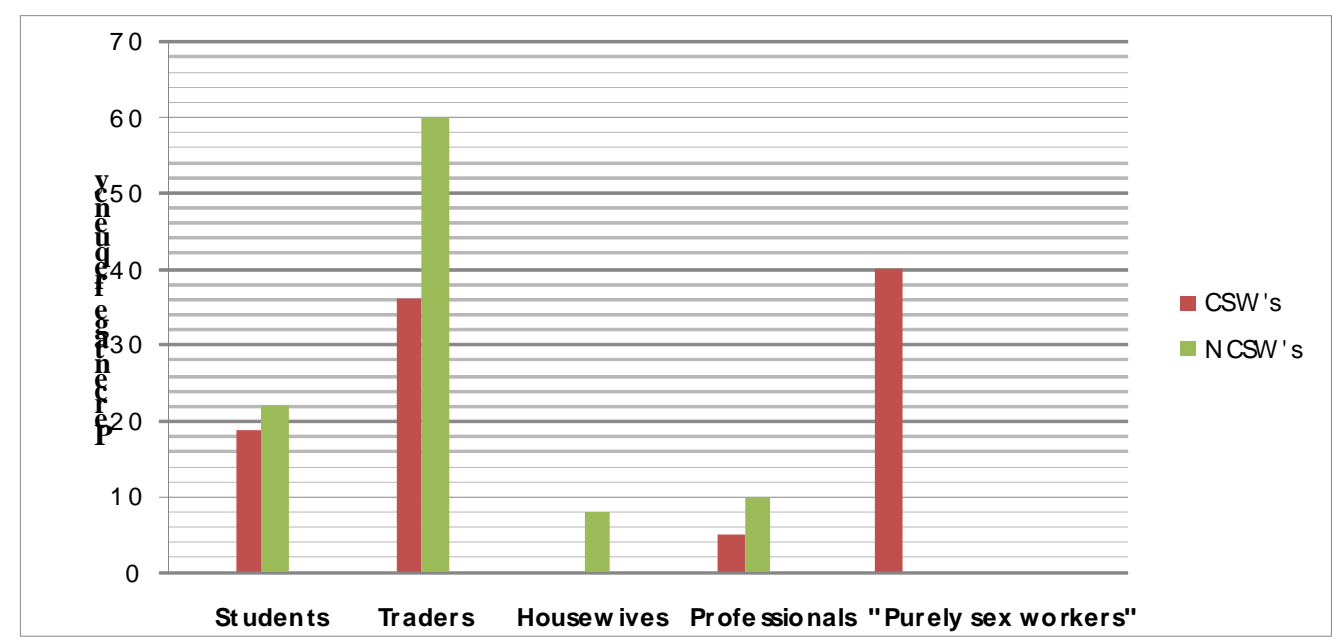




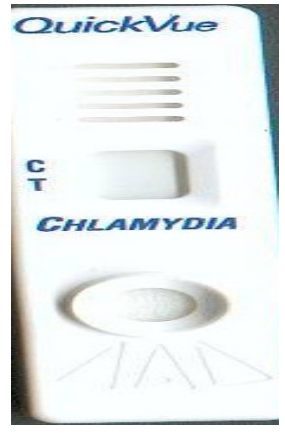

I

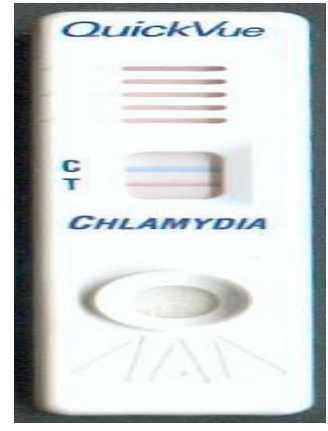

II

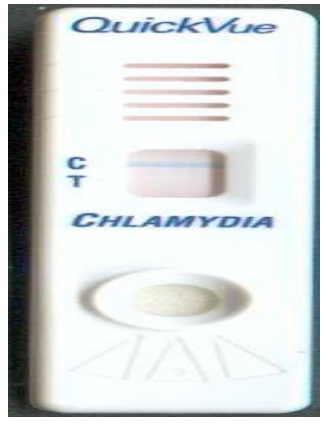

III

Plate.1 Photograph showing QuickVue Chlamydia test outcomes

I - Uninoculated Cassette; II - Positive Case; two visible bands

III - $\quad$ Negative Case one visible band; $\mathrm{C}-$ Band $=$ Control Band; $\quad$ T- Band $=$ Test Band

Gonorrheal infection was found in only $1 \%$ of the CSWs and none at all in the nonCSWs. Gonorrhoea used to be a common sexually transmitted infection some decades ago. Its incidence is now on the decline, perhaps, as a result of potent antimicrobial agents that have been used in its management. Additionally, there has been an increase public education on the causes, symptoms, management and prevention of the gonorrhoea among Ghanaians. When an educational or a control programme is implemented and properly followed the incidence of diseases on which the programme is intended for reduces. For example, during 1975 - 1997, the national gonorrhea rate in the United States of America declined by $74 \%$ after implementation of the national gonorrhea control program (CDC, 2012).

The study revealed that most of the CSWs were aged between 18 to 22 years, followed by 23 to 27 years; a total of $85 \%$ as seen in Table 1 . Relatively young women are more prone to engaging in commercial sex; at these ages they are strong, more attractive to men, and can sexually satisfy their clients. Many studies have confirmed that most CSWs entered into harlotry before age eighteen. For example, Sister Speak Out study of 222 women in prostitution in Chicago showed that $35 \%$ entered prostitution before age of $15 ; 62 \%$ before age 18 , and $87 \%$ entered before attaining the age of 21 (Jody and Shapiro, 2002). Men who patronize services of CSWs also prefer such age bracket more than the 'older' women. According to Berarovich, 2014, things men look out for when hiring escorts are much more complex and they include youthfulness as well as beauty. The demand for services of the CSWs is more skewed towards the younger ones.

It also came to light, from the study that $41 \%$ of the CSWs did not have any formal education (results not shown). Illiteracy may fuel prostitution out of ignorance. Most of this category of CSWs may have entered out of pure ignorance of the risks involved in the job they are engaged in. They were probably lured into harlotry by poverty as without education there is less probability of getting a decent formal job to do. Women, ranging from students to those in various jobs also engage in commercial sex work, perhaps, to supplement their incomes.

The study has shown that Chlamydia infection is higher among commercial sex workers in the Kumasi Metropolis. The 
disturbing aspect of the high prevalence rate of the infection is the fact that those having it did not know that they are carrying such an infection as they are asymptomatic. Treatment, though are available and accessible, they would not seek as they do not show any symptoms of the disease, and would therefore continue to transfer to others.

\section{Acknowledgement}

The authors wish to thank Dr. Baffour Poku and all the staff at the Microbiology laboratory at KATH and also Dr. Agyarko Poku at the STI Clinic of Suntreso Hospital all in Kumasi for their support in diverse ways.

\section{References}

Anttila T., Saikku P., Koskela P., Bloigu A., Dillner J., Ikaheimo I., Jellum E., Lehtinen M., Lenner P., Hakulinen T., Narvanen A., Pukkala E., Thoresen S., Youngman L. and Paavonen J. 2001. Serotypes of Chlamydia trachomatis and risk for development of cervical squamous cell carcinoma. JAMA; 28:547-51.

Apea-Kubi K.A., Yamaguchi S., Sakyi B., Kishimoto T., Ofori-Adjei D., Hagiwara T. 2004. Neisseria gonorrhoea, Chlamydia trachomatis and Treponema pallidum infections in antenatal and gynecological patients at the Korle-Bu Teaching Hospital, Ghana. Jpn J Infect Dis; 57 (6): 253-6.

Berarovich C. 2014. Harlotry: The Retirement Age For A Hooker Isn't As Young As You Think. 'http://www.thegloss.com/2014/05/20 i/sex-and-dating/harlotry-retirement'age-hooker-isnt-young-1 think/\#ixzz3fu3nKpca. Accessed on $10^{\text {th }}$ July, 2015.

Bolam M.J, Stokes T., Shukla R., Bhaduri S. and Schober P. 1997. Controlling genital Chlamydia infection. BMJ; 314 (7072): 516-516.

Buve A., Weiss H.A. and Laga M. 2001. The epidemiology of Gonorrhoea, Chlamydia infections and syphilis in four African cities. AIDS; 15 (Suppl 4): 579.

CDC (Centre for Disease Control) 2012. Sexually Transmitted Diseases Surveillance. hittp://www.cdc.gov/std/stats12/gono rrhea.htm. Accessed on $2^{\text {nd }}$ July, 2015.

CDC 2014. Sexually Transmitted Infection: A Major Women's Health Concern. ihttp://www.rhtp.org/std/types.asp. Accessed on $9^{\text {th }}$ May, 2014.

CDCP (Centers for Disease Control and Prevention) 1993. Recommendations for the prevention and management of Chlamydia trachomatis infections, 1993. Morb. Mortal. Wkly. Rep. Recomm. Rep. 42:1-39.

Coombes R. 2004. Doctors demand national screening for Chlamydia. BMJ; 328:1397-0.

Drescher C, Elkins TE, Adkeo O, AkinsBekoe P, Agbemadzo T, Foster RL, Martey J O. 1988. The incidence of urogenital Chlamydia trachomatis infections among patients in Kumasi, Ghana. Int J Gynaecol Obstet.; 27 (3): 381-3.

Jody R. and Shapiro D.L. 2002. Sisters Speak Out: The Lives and Needs of Prostituted Women in Chicago. A Research Study. Center for Impact Research, August 2002.

Mishori R., Mcclaskey E.R, and Winklerprins V.J. 2012. Chlamydia Trachomatis Infections: Screening, Diagnosis, and Management. Am Fam Physician. 86(12):1127-1132. 
Ngandjio A., Clerc M., Fonkoua M.C., Thonnon J., Njock F., Pouillot R., Lunel F. Bebear C., de Barbeyrac B. and Bianchi A. 2003. Screening of volunteer students in Yaounde, Cameroon, Central Africa for Chlamydia trachomatis infection and Genotyping of isolated Chlamydia trachomatis strain. J Clin Microbiol; 41(9): 4404-4407.

Sahoo C.K., Tamhankar A.J., Johansson E. and Lundborg, S.C. 2010. Antibiotic use, resistance development and environmental factors: a qualitative study among healthcare professionals in Orissa, India. BMC Public Health 2010, 10:629-638.

Simms I, Hughes G., Catchpole M 1998. Screening for Chlamyida trachomatis. BMJ; 317 (713-9): 680630.

Thayer, J.D. and Martin, J.E. Jr. 1966. Improved medium selective for cultivation of $N$. gonorrhoeae and $N$. meningitidis. Public Health Rep. 81:559.

\section{How to cite this article:}

Amina Abubakari, Samuel N. Osei-Djarbeng, John Asiedu Larbi and Enoch H. Frimpong. 2016. Presence of Chlamydia Infection among asymptomatic Female Commercial Sex-workers (CSWs) in the Kumasi Metropolis, Ghana. Int.J.Curr.Microbiol.App.Sci. 5(1): 342-349 http://dx.doi.org/10.20546/ijcmas.2016.501.032 